\title{
Cortisol salival en niños con y sin bruxismo
}

\section{Resumen}

Objetivo: evaluar y comparar valores de facetas de desgaste y de cortisol salival en niños con y sin bruxismo posible y con bajos y altos niveles de inestabilidad emocional. Métodos: Se recolectaron datos en 17 niños sanos con bruxismo posible (B) según la lista de comportamientos orales de los Criterios Diagnósticos para Trastornos Temporomandibulares, diferenciando bruxismo de sueño (BS) y de vigilia (BV). Se aparearon por género y edad con un grupo control, sin reporte de bruxismo (C). Cinco odontopediatras identificaron facetas de desgaste con el índice de desgaste dentario (IDD) (kappa interobservador 0,94). El dosaje de cortisol se realizó con saliva completa matinal mediante electro-quimio-luminiscencia. La inestabilidad emocional (Ie) se evaluó en los mayores de 8 años con el cuestionario "Big Five" de personalidad para niños y adolescentes. Resultados: La edad media fue $11,3 \pm 2,7$, con $59,6 \%$ de género femenino. En el grupo de bruxismo;
Silvina Gabriela Cortese, ${ }^{1}$ Ingrid Clarisa Guitelman, ${ }^{2}$ Ana María Biondi. ${ }^{3}$
$35,3 \%$ reportó BS, 35,3\% BS + BV y $29,4 \%$ BV. No se hallaron diferencias en el desgaste promedio para dientes primarios $(p=0,3858)$ ni para permanentes $(p=0,7478)$ entre ambos grupos. La media de cortisol salival fue $0,181 \pm 0,183$ para el grupo de estudio y 0,183 $\pm 0,161$ para el grupo control $(p=0,9863)$. Para la Inestabilidad emocional baja la media de niveles de cortisol fue $0,142 \pm 0,08 \mathrm{ug} / \mathrm{dl}$ y en Ie alta $0,149 \pm 0,07$ ( $p=0,0437)$. Todos los pacientes con BS + BV, presentaron inestabilidad emocional alta $(p=0,006)$. Conclusiones: El desgaste en ambas denticiones no mostró diferencias significativas. Los valores de cortisol salival obtenidos en este estudio no permitirían considerarlo factor relevante para el diagnóstico de bruxismo. En condiciones de inestabilidad emocional extremas se hallaron diferencias en los niveles de cortisol salival y en la frecuencia que reportan ambos tipos de bruxismo.

Palabras clave: saliva, bruxismo, niños, cortisol, inestabilidad emocional.

\footnotetext{
1.Profesora Asociada. Facultad de Odontología, Cátedra Odontología Integral Niños, Universidad de Buenos Aires, Buenos Aires, Argentina.

2. Jefe de Trabajos Prácticos. Facultad de Odontología, Cátedra Odontología Integral Niños, Universidad de Buenos Aires, Buenos Aires, Argentina.

3. Profesora Titular. Facultad de Odontología, Cátedra Odontología Integral Niños, Universidad de Buenos Aires, Buenos Aires, Argentina.
} 
Artigo original

\section{Cortisol salivar em crianças com ou sem bruxismo}

\section{Resumo}

Objectivo: Avaliar e comparar valores de facetas de desgaste e de cortisol salivar em crianças com ou sem provável bruxismo e com baixos e altos níveis de instabilidade emocional. Métodos: Foram coletados dados em 17 crianças saudáveis com provável bruxismo (B) segundo a lista de comportamentos orais (OBC) do Critérios Diagnósticos para Desordem Temporomandibular, diferenciando bruxismo do sono (BS) e de vigília (BV). As crianças foram agrupadas em duplas, por género e idade como um grupo controle, sem informação de bruxismo (C). Cinco Odontopediatras identificaram facetas de desgaste com o índice de desgaste dentário IDD (Kappa interobservador 0,94). A dosagem de cortisol se realizou com saliva completa matinal mediante eletro-quimio-luminiscencia. A instabilidade emocional foi avaliada nos maiores de 8 anos com o questionário de personalidade BFQ-NA. Resultados: Idade média $11,3 \pm 2,7 ; 59,6 \%$ gênero feminino. No grupo bruxismo, 35,3\% reportaram BS, $35,3 \%$ BS+BV e $29,4 \%$ BV. Não foram encontradas diferenças quanto ao desgaste médio para dentes primários $(\mathrm{p}=0,3858)$ nem para permanentes $(p=0,7478)$ entre os dois grupos. A média de cortisol salivar foi de 0,181 $\pm 0,183$ no grupo de estudo e $0,183 \pm 0,161$ em no grupo de controle $(p=0,9863)$. Com instabilidade emocional baixa, a média de níveis de cortisol foi de $0,142 \pm 0,08 \mathrm{ug} / \mathrm{dl}$ e em alta $0,149 \pm 0,07$ $(p=0,0437)$. Todos os pacientes com BS+BV apresentaram instabilidade emocional alta $(p=0,006)$. Conclusões: $O$ desgaste em ambas dentições não mostrou diferenças significativas. Os valores de cortisol salivar obtidos no presente estudo não permitiram considerá-lo como fator relevante para o diagnóstico. Em condições de instabilidade emocional extrema, foram encontradas diferenças nos níveis de cortisol salivar e na frequência que reportam os dois tipos de bruxismo.

Palavras chaves: Saliva, bruxismo, criança, cortisol, instabilidade emocional.

Original article

\section{Salivary cortisol in bruxing and non-bruxing children}

\section{Abstract}

Objective: To evaluate and compare the degree of tooth wear and salivary cortisol levels in bruxing and non-bruxing children with low and high levels of emotional instability. Methods: Data were collected from a sample of 17 healthy children with possible bruxism (B) according to the Oral Behaviors Checklist (OBC) of the Diagnostic Criteria for Temporomandibular Disorders differentiating sleep (BS) and wakefulness 
(BV) bruxism. They were matched by sex and age to a control group without report of bruxism (C). Five pediatric dentists calibrated for the tooth wear index (TWI) (kappa interobserver 0.94) registered the occurrence of incisor/occlusal tooth wear (probable bruxism). The dosage of cortisol was performed in a sample of morning saliva by electrochemiluminescence. Emotional instability was measured by the BFQ Big Five Questionnaire for Children (for children older than 8 years of age). Results: Sample's mean age was: $11.3 \pm 2.7,59.6 \%$ were female. In bruxism group $35.3 \%$ reported BS, $35.3 \%$ BS + BV and $29.4 \%$ BV. No differences were found between both groups for tooth wear in primary $(p=0.3858)$ and permanent dentition ( $p=0.7478)$. In the study group average of cortisol level was

\section{Introducción}

En 2013, fue publicado un consenso internacional por un grupo de expertos con el objetivo de proponer la definición y sistema de clasificación de diagnóstico para el bruxismo que pudieran ser adoptados por investigadores, y profesionales clínicos. En este sentido el bruxismo fue definido como una actividad repetitiva músculo-mandibular caracterizada por apretar o rechinar los dientes y/o propulsar la mandíbula, presentando dos diferentes manifestaciones circadianas: del sueño y de la vigilia. Denominaron Bruxismo "posible" al diagnosticado solo a través del auto-reporte, "probable", cuando se complementa con el examen clínico y "definitivo" cuando se completa con estudios tales como polisomnografía y/o electromiografía. ${ }^{1}$ Esta nueva clasificación surgió como consecuencia de la subjetividad y de la inexactitud de
$0.181 \pm 0.183$ and $0.183 \pm 0.161$ in control group $(p=0.9863)$. Mean cortisol level was $0.142 \pm 0.08 \mathrm{ug} / \mathrm{dl}$ for low emotional instability, and $0.149 \pm 0.07$ for high $(\mathrm{p}=0.0437)$. All the patients who had both $\mathrm{SB}$ and $\mathrm{AB}$ were in the high emotional instability group $(p=0.006)$. Conclusions: Tooth wear in both dentitions showed no differences. Results obtained suggest that the dosage of salivary cortisol would not be relevant as a biomarker for the suggestion of bruxism in children. Patients exhibiting extreme conditions of emotional instability, showed significant differences in salivary cortisol levels and in the frequency they reported both types of bruxism.

Key words: saliva, bruxism, child, cortisol, emotional instability.

las respuestas que a veces revelan los cuestionarios de auto-reporte y porque el desgaste dentario muestra efecto acumulativo así como dificultad para realizar el diagnóstico diferencial con otras entidades tales como la erosión.

En la clínica cotidiana con niños, solo es factible trabajar con "bruxismo probable", siendo inadmisible realizar los estudios complementarios propuestos, tanto por la complejidad como por costo, solo con fines de diagnóstico odontológico de un bruxismo definitivo.

Si bien se acepta que la etiología es multifactorial, autores como Lobezoo et $a .^{2}$ y Manfredini et al. ${ }^{3}$ sugirieron un cambio conceptual, considerando que el bruxismo está regulado por factores centrales y su actividad modulada por neurotransmisores. En 1999 Vanderas et al. concluyeron que los niveles de dopamina 
y epinefrina en orina de 24 horas de niños de 6 a 8 años se encontraban fuertemente asociados al bruxismo reconocido por cuestionario y examen clínico. ${ }^{4}$

Actualmente algunos estudios analizaron los niveles de cortisol salival en pacientes con bruxismo ${ }^{5,6}$ siendo la recolección de muestra salival un procedimiento no invasivo, seguro y fácilmente repetible. Puede realizarse fuera del ámbito del laboratorio, tantas veces como fuera necesario, siendo la saliva un instrumento útil con fines diagnósticos viable en pacientes pediátricos. ${ }^{7}$

Por otra parte Castelo et al. ${ }^{8}$ utilizaron cuestionarios y presencia de facetas en dientes permanentes como criterios para el diagnóstico de bruxismo en un grupo de niños con dentición mixta, pero reconocen no haber evaluado ansiedad, registrada anteriormente por Monaco et al. ${ }^{9}$ quienes concluyeron que los datos obtenidos en su estudio respaldan el concepto de que el estado de ansiedad es un factor prominente en el desarrollo del comportamiento del bruxismo en los niños.

La prueba psicométrica "Big Five" $(B F Q-N A)^{10}$ con nivel de aplicación en niños de 8 a 15 años, permite evaluar las cinco dimensiones de la personalidad: inestabilidad emocional, extraversión, apertura, amabilidad y conciencia. Presenta niveles aceptables de validez y fiabilidad. El factor inestabilidad emocional evalúa la tendencia al malestar y al neuroticismo, manifestados en los cambios de humor, la tendencia a la tristeza, ansiedad e irritabilidad.

En un trabajo realizado con anterioridad en la Cátedra de Odontología Integral Niños (OIN) de la Facultad de Odontología de la Universidad de Buenos Aires (FOUBA) sobre aspectos de la personalidad, hábitos, disfunción de la Articulación TémporoMandibular (ATM )en pacientes con y sin bruxismo se encontró en bruxistas escasa frecuencia de valores bajos de inestabilidad emocional. ${ }^{11}$

Durante más de 20 años se utilizaron los Criterios Diagnósticos para Investigación de Trastornos Témporomandibulares (CDI/TTM $){ }^{12}$ cuyo eje I sobre condiciones clínicas incluye 3 preguntas con 2 opciones sobre bruxismo. Actualmente se estableció una segunda versión recomendada tanto para uso clínico como investigación $(\mathrm{CD} / \mathrm{TTM})^{13}$ que incrementó la sensibilidad y especificidad, incluyendo una lista de evaluación de comportamientos orales (OBC), aumentando el número de preguntas en relación a la actividad diurna, además de cuantificar la frecuencia mediante una escala Likert.

Los factores cognitivos y de comportamiento, como ansiedad y estrés, generan respuestas hormonales, probablemente debido a la estimulación glandular. El cortisol, se describe como posible indicador de ansiedad, estrés y depresión. ${ }^{14}$

Los objetivos de este trabajo fueron evaluar y comparar los valores de dosaje de cortisol salival y la presencia de facetas de desgaste en niños con y sin bruxismo posible, así como comparar los niveles de cortisol salival en niños con bajos y altos niveles de inestabilidad emocional con y sin bruxismo reportado. 


\section{Materiales y Métodos}

El diseño del estudio fue de tipo prospectivo caso/control, aprobado por el Comité de ética de la FOUBA (UBACYT 20720150200023BA).

La muestra se conformó con pacientes de ambos géneros de 5 a 15 años que concurrieron para su atención a la Cátedra Odontología Integral Niños y brindaron asentimiento y sus acompañantes consentimiento. Fueron excluidos los niños con problemas del desarrollo, compromiso médico, trastornos neurológicos, y psiquiátricos, y quienes se encontraban realizando tratamientos de ortopedia/ortodoncia así como también quienes presentaban lesiones bucales sangrantes o que utilizaran esteroides exógenos dado que interfieren el test salival.

\section{Bruxismo Posible}

Los datos se recolectaron entre marzo y junio de 2017, en un grupo de 17 niños sanos con bruxismo posible (B) que respondieron con ayuda de sus padres, el cuestionario de la lista de comportamientos orales $(\mathrm{OBC})$ del CD/TTM, diferenciando bruxismo de sueño (BS) y de vigilia (BV). Se aparearon por género y edad con un grupo control del mismo servicio, sin reporte de bruxismo $(\mathrm{C})$.

\section{Bruxismo Probable}

Integraron el equipo profesional 5 odontopediatras calibradas en el índice de desgaste dentario (IDD) ${ }^{15}$ (kappa interobservador 0,94$)$ quienesidentificaron clínicamente las facetas de desgaste de toda la dentición presente (bruxismo probable) en sillón odontológico, con luz de foco, previa profilaxis con cepillado mecánico, enjuague y dientes secos, utilizando una planilla ad hoc para registrar cada diente en las zonas de contacto con antagonistas: $0=$ esmalte intacto, $1=$ faceta en esmalte, 2 = faceta en dentina, 3 = faceta en dentina profunda, realizando además el registro fotográfico con definición de $5 \mathrm{MP}$ a $15 \mathrm{~cm}$ de distancia focal al arco dentario. De cada paciente se obtuvieron separadamente el desgaste promedio para dientes primarios y permanentes.

\section{Cortisol salival:}

La recolección de muestras de saliva completa se realizó en un tubo Salivette ${ }^{\circledR}$ Sarstedt, entre las 11.30 y 12.30 hrs., luego de una hora de haber realizado la profilaxis dental sin que hubiera ingerido alimentos y bebidas, considerando las fluctuaciones diurnas del cortisol (Figura 1). Fueron almacenadas en heladera a $4^{\circ} \mathrm{C}$ hasta ser entregadas al laboratorio de la Cátedra de Bioquímica General y Bucal, donde se centrifugaron a $2.500 \mathrm{rpm}$ durante $10 \mathrm{~min}$ almacenando el sobrenadante a $-40{ }^{\circ} \mathrm{C}$ hasta su procesamiento mediante electro-quimioluminiscencia en un laboratorio de análisis clínicos hormonales.

Inestabilidad emocional

Se administró solo a los niños mayores de 8 años el cuestionario "Big Five" de personalidad para niños y adolescentes (8 a 15 años) (BFQ-NA), que consta de:

a) Un ejemplar con 65 ítems, en escala Likert (con 5 opciones que van desde "casi siempre" a "casi nunca") a completar por el niño 


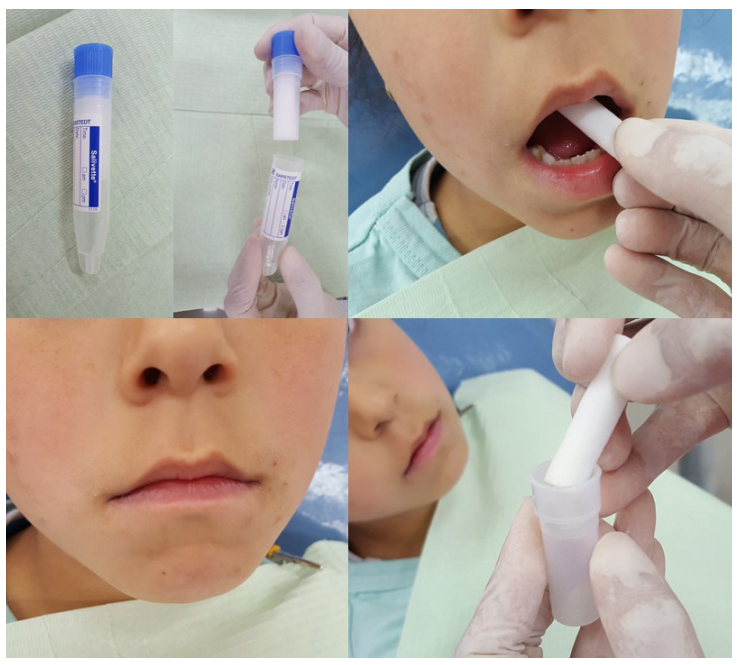

Figura 1. Secuencia de recolección de saliva.

b) Un manual que el evaluador utiliza para la corrección e interpretación de la prueba.

c) Un código de tarjeta que se emplea para realizar la corrección en líneas de la prueba.

El test fue respondido en forma individual por cada paciente sin distracciones y en silencio, siendo el tiempo medio necesario entre 25 y 30 minutos.

Se comprobó que todos los ítems fueran contestados, ya que la presencia de respuestas en blanco disminuye sensiblemente la fiabilidad y validez de la prueba. En el caso que se hubiese omitido una respuesta, se instó al paciente a que la respondiera. Por este motivo el sistema de corrección de la prueba incluye el control del número de respuestas en blanco, si se detectan más de seis, invalida el caso y no ofrece puntuaciones del sujeto.

Los resultados fueron obtenidos en forma automática por sistema en línea en donde se siguieron las instrucciones que presentaba la hoja con el código de tarjeta para la corrección. Para ello, una vez en el sistema, se introdujeron todas las respuestas dadas por el paciente realizando el sistema la corrección y puntuación de los distintos factores, y ofreciendo un perfil gráfico con las puntuaciones $\mathrm{T}$ de cada factor de personalidad del paciente. Éstas constituyen una escala típica de unidad constante, con una media en el valor 50 y una desviación de 10 puntos.

Luego de procesar los datos de cada test, se cargaron en una base de datos los resultados de las puntuaciones $\mathrm{T}$ de los registros de Inestabilidad emocional (Ie), los valores del desgaste y los niveles de cortisol de cada paciente.

Análisis estadístico:

Para la obtención de los resultados se utilizaron porcentajes, medias, desviaciones estándar y comparaciones mediante test de Mann-Whitney, Chi cuadrado (Yates) y ANOVA (Tukey)

\section{Resultados}

La edad media del grupo fue 11,3 $\pm 2,7$ años, perteneciendo el 59,6 \% de la muestra al género femenino.

\section{Bruxismo}

En el grupo de Bruxismo (B) el 35,3\% reportó bruxismo del sueño (BS), 35,3\% sueño y vigilia (BS + BV) y 29,4 \% solo vigilia (BV).

El desgaste promedio reportado, considerando la unidad paciente, se 
expresa en la Tabla 1, sin hallazgos de diferencias significativas para dientes primarios, $(p=0,3858)$ ni para permanentes $(p=0,7478)$

Tabla 1. Valores promedio y desviación estándar de desgaste en ambas denticiones.

\begin{tabular}{|lcc|}
\hline & $\begin{array}{c}\text { Grupo } \\
\text { Bruxismo (B) }\end{array}$ & $\begin{array}{c}\text { Grupo } \\
\text { Control (C) }\end{array}$ \\
\hline $\mathrm{N}=$ & 17 & 17 \\
\hline $\begin{array}{l}\text { Desgaste } \\
\text { dentición } \\
\text { primaria }\end{array}$ & $1,24 \pm 0,57$ & $0,92 \pm 0,54$ \\
\hline $\begin{array}{l}\text { Desgaste } \\
\text { dentición } \\
\text { permanente }\end{array}$ & $0,54 \pm 0,27$ & $0,28 \pm 0,32$ \\
\hline
\end{tabular}

Cortisol

En relación al cortisol salival, todos los valores hallados se encontraban dentro de los parámetros de normalidad para cortisol matinal, (0,101-1,330 ug/dl).

En B la media fue 0,181 $\pm 0,183$ y $0,183 \pm 0,161$ en $C(p=0,9863)$. Tampoco se hallaron diferencias analizando separadamente BS $(p=0,7733)$ y BV
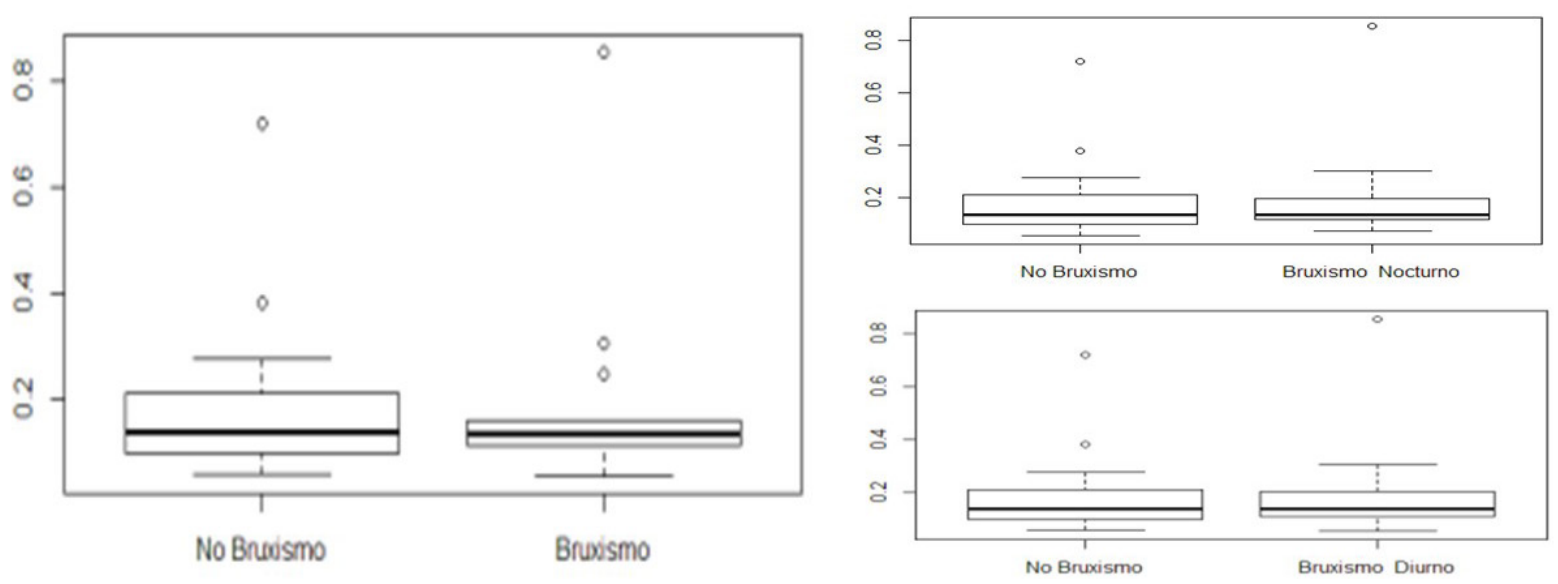

Figura 2: Boxplots que grafican la dispersión de cortisol en los diferentes grupos.

$(p=0,7241)$. En los boxplots (Figura 2) se observa que la menor dispersión se expresó en el grupo con bruxismo posible, aunque los valores medios de todos los grupos fueron semejantes.

En la Tabla 2 se describen los valores promedio según el tipo de bruxismo reportado.

\section{Inestabilidad emocional}

El análisis se realizó en 14 niños del grupo conbruxismo posibley 14 del grupocontrol, excluyendo de cada grupo a 3 menores de 8 años, por no ser aplicable la prueba. Se conformaron 2 grupos: IeB $(n=14)$ con puntuaciones bajas <44, y IeA $(n=9)$ con altas $>56$, excluyendo para el análisis de los resultados las correspondientes a los de valores medios (Figura 3). La edad media fue 12 años $+0,14$. Entre IeB y IeA no hubo diferencias en relación al género, la edad, el BS y el BV ( $>>0,05)$. En IeB la media de niveles de cortisol fue $0,142 \mathrm{ug} / \mathrm{dl}$ DS 0,08 y en IeA 0,149 DS 0,07 ( $p=0,0437)$. Todos los pacientes que presentaron simultáneamente $\mathrm{BS}$ y $\mathrm{BV}$, pertenecían a IeA $(p=0,006)$ (Tabla 3). 
Tabla 2. Dosajes promedio de cortisol y promedios de desgaste de incisivos y primeros molares permanentes de los pacientes con diferentes tipos de bruxismo.

\begin{tabular}{|lccccc|}
\hline & N $=$ & Edad & Cortisol ug/dl & Fac. Inc Perm & Fac. Molares Perm \\
\hline Sueño & 6 & $9,8 \pm 2,9$ & $0,125 \pm 0,025$ & $0,7 \pm 0,4$ & $0,95 \pm 0,1$ \\
\hline Vigilia & 5 & $13 \pm 0,9$ & $0,117 \pm 0,039$ & $0,8 \pm 0,3$ & $0,45 \pm 0,4$ \\
\hline Ambos & 6 & $11,3 \pm 2,5$ & $0,291 \pm 0,262$ & $0,37 \pm 0,34$ & $0,41 \pm 0,45$ \\
\hline
\end{tabular}

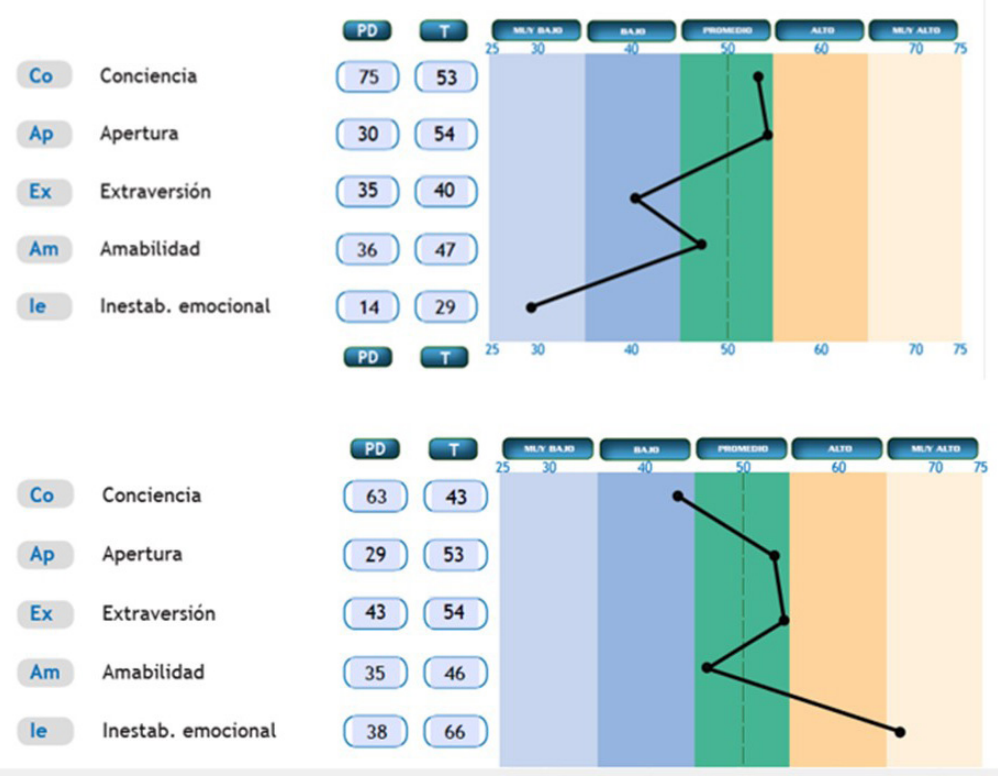

Figura 3. Resultados de 2 pacientes del BFQ-NA para los cinco factores de personalidad, donde se observan valores de Ie bajo y alto.

Tabla 3. Comparación de diferentes variables entre IeB - IeA

\begin{tabular}{|lccc|}
\hline & IeB $(<44)$ & IeA $(>56)$ & $\mathrm{p}=$ \\
\hline $\mathrm{N}=$ & 14 & 9 & \\
\hline Masculinos & 3 & 5 & 0,09 \\
\hline Edad & $12,6 \pm 1,3$ & $12,1 \pm 1,6$ & \\
\hline $\begin{array}{l}\text { Cortisol } \\
\mu \mathrm{g} / \mathrm{dl}\end{array}$ & $0,142 \pm 0,08$ & $0,149 \pm 0,07$ & 0,0437 \\
\hline $\mathrm{BV}$ & 3 & 1 & 0,52 \\
\hline $\mathrm{BS}$ & 3 & 1 & 0,52 \\
\hline $\mathrm{BV}+\mathrm{BS}$ & 0 & 4 & 0,006 \\
\hline Ie promedio & $42,5 \pm 9,7$ & $61,5 \pm 4,13$ & \\
\hline
\end{tabular}

\section{Discusión}

El diagnóstico y la etiología del bruxismo en niños son aún más controvertidos que en adultos. ${ }^{17,18}$ La mayoría de los estudios hacen foco solo en el bruxismo nocturno y la confirmación mediante polisomnografías, considerado el método de diagnóstico gold standard. ${ }^{19}$ Los cuestionarios para identificar bruxismo posible tienen una confiabilidad aceptable pero aún presentan algunos inconvenientes. En principio son solo aplicables a mayores de 8 años, razón por la cual, en este trabajo el análisis de la Inestabilidad emocional pudo ser realizado solamente en 14 parejas de las 
17 que integraron la muestra. El CDI/TMD usado anteriormente contenía 3 preguntas con 2 opciones de respuestas (positivo o negativo). El protocolo actual $\mathrm{OBC}$ del CD/TMD, utilizado en este estudio amplió las opciones a 5 con escala Likert según la frecuencia de las conductas. El consorcio Internacional de IADR, autor de ambos instrumentos de evaluación, publicó recientemente el Manual de puntuación para instrumentos de auto-reporte, donde destaca que aún no se han establecido las propiedades de medición de la lista de verificación de comportamientos orales con el fin de determinar mejor la presencia de comportamientos parafuncionales. ${ }^{20}$ Restrepo refiere que en relación al bruxismo nocturno mayoritariamente los padres duermen alejados de los niños, sin poder aportar información fehaciente, situación que podría verse reflejada en este trabajo, en los niños cuyos auto-reportes expresan no bruxar, pero que sin embargo mostraron facetas de desgaste en dientes permanentes. ${ }^{19}$

Respecto a la confirmación de bruxismo probable mediante la identificación de facetas, tampoco es concluyente, ya que el bruxismo por apretamiento no produce desgaste. En un trabajo anterior sobre análisis del desgaste dentario en niños de 10 a 15 años $^{21}$ donde se utilizó el CDI/ TTM y una muestra de mayor tamaño se observaron diferencias entre las facetas en dentición permanente entre ambos grupos $(0,41 \pm 0,5 ; 0,36 \pm 0,47 \mathrm{p}=0,003)$.

Por estas razones surge la necesidad de encontrar indicadores diagnósticos sencillos y fiables, que sumados a la teoría de factores centrales psicosociales como estrés percibido, ansiedad y características de la personalidad y fisiopatológicos como reguladores del bruxismo, fueron promotores de investigaciones sobre bruxismo y cortisol salival.

Existe evidencia que avala el uso de cortisol salival como determinación diagnóstica, ya que se correlaciona de manera predecible con el cortisol total, siendo su concentración independiente de la tasa de flujo. ${ }^{22}$

El aumento del cortisol al despertar tiene carácter genético, mientras que la variabilidad en el descenso a lo largo del día se relaciona con influencias ambientales. En condiciones fisiológicas estables, este ritmo se presenta con regularidad, sin verse afectado por la edad, el género, la etapa de desarrollo o la composición corporal. ${ }^{23}$ En este estudio todas las muestras fueron recolectadas entre las 11.30 y las 12.30 horas y fueron procesadas mediante el método automatizado EQL.

Karakoulaki et al. en $2015^{5}$ hallaron correlación entre los niveles de cortisol y bruxismo nocturno en adultos. En 2012 Castelo, $^{8}$ en un grupo de niños que reportaron bruxismo nocturno no encontró variaciones en los niveles matinales de cortisol, concordando con los resultados de este estudio. Si bien Amato et al. ${ }^{24}$ reportaron bruxismo nocturno $y$ altos niveles de cortisol en niños asmáticos, también expresan que no se puede confirmar relación directa entre el bruxismo y los niveles de cortisol salival.

La conducta implica una interacción entre la personalidad subyacente de una persona y las variables situacionales. En la mayoría de los casos, las respuestas son coherentes con los rasgos de personalidad subyacentes. La inestabilidad emocional es un rasgo de personalidad que conlleva, ansiedad, mucha preocupación, $\mathrm{y}$ 
percepción sesgada hacia las situaciones negativas. ${ }^{10}$ Se caracteriza por la falta de homogeneidad en la conducta, baja tolerancia al estrés, poca sociabilidad, nerviosismo, inseguridad e hipocondría.

Las investigaciones entre la asociación del bruxismo y estrés proporcionaron resultados contradictorios ${ }^{2,5}$ que pueden ser atribuidos a la naturaleza multifactorial del bruxismo, diferentes metodologías para la evaluación del estrés, diferentes tipos de biomarcadores en saliva relacionados al estrés y a la divergencia de la definición de estrés como un riesgo o factor etiológico. ${ }^{5}$

Oliveira et al. en 2015 concluyeron que existe una relación directa entre la presencia de un desorden de ansiedad y el comienzo del bruxismo en niños. También revelaron que los niños con bruxismo tenían altos niveles en la escala STAIC (the State-Trait Anxiety Inventory for Children) comparados con los no bruxistas. ${ }^{25}$

Los resultados de Kobayashi et al. ${ }^{26}$ no revelaron diferencias en cuanto a la secreción salival asociada a parámetros de stress entre grupos de niños con y sin TTM, mientras que Castelo et al. ${ }^{8}$ hallaron que los niveles de cortisol en pacientes con bruxismo eran menores que aquellos sin bruxismo.

En el presente estudio se encontraron diferencias significativas en cuanto a la media en los niveles de cortisol en niños con bajos y altos niveles de inestabilidad emocional resultados que concuerdan con los obtenidos en otros trabajos donde se encontró relación positiva entre ansiedad y niveles de cortisol alto. ${ }^{5,27}$ Es importante destacar que si bien no se encontraron diferencias en los niveles de cortisol entre BS y BV entre ambos grupos, todos los pacientes que presentaron simultáneamente BS y BV, pertenecían al grupo con mayores valores de inestabilidad emocional. Una revisión sistemática realizada por Cruz en $2016{ }^{28}$ revela que la evidencia no es concluyente entre los niveles de cortisol salival y bruxismo, refiriendo la necesidad de continuar las investigaciones.

\section{Conclusiones}

Los valores de cortisol salival hallados en esta muestra se encuentran dentro los valores matinales de normalidad, con amplia variabilidad individual. No se encontraron diferencias, entre el grupo con bruxismo y el grupo control que permita considerarlo un factor relevante para el diagnóstico, en sus diferentes tipos. El grado de desgaste en ambas denticiones tampoco mostró diferencias significativas entre los grupos. En condiciones de inestabilidad emocional extremas se hallaron diferencias en los niveles de cortisol salival y en la frecuencia que reportan ambos tipos de bruxismo. Se requieren otros estudios para profundizar la relación entre las variables que ayuden a esclarecer el bruxismo en niños.

\section{Agradecimientos:}

A las odontólogas Melisa Ienco y Diana Fridman, integrantes del proyecto y a la Lic. Lucía Babino por la colaboración en el procesamiento estadístico.

Financiamiento:UBACyT20720150200023BA. Los autores no presentan conflictos de interés 


\section{Referencias bibliográficas}

1. Lobbezoo F, Ahlberg J, Glaros A G, et al. Bruxism defined and graded: an international consensus. J Oral Rehabil 2013; 40: 2-4.

2. Lobbezoo F, Naeije M. Bruxism is mainly regulated centrally, not peripherally. J Oral Rehabil 2001; 28: 1085-91.

3. Manfredini D, Landi N, Fantoni F et al. M. Anxiety symptoms in clinically diagnosed bruxers. J Oral Rehabil 2005; 32: 584-8.

4. Vanderas AP, Menenakou M, Kouimtzis T et al. Urinary catecholamine levels and bruxism in children. J Oral Rehabil. 1999; 26: 103-10.

5. Karakoulaki S, Tortopidis D, Andreadis D et al. Relationship between sleep bruxism and stress determined by saliva biomarkers. Int J Prosthodont 2015; 28: 467-74.

6. Allwood MA, Handwerger K, Kivlighan KT et al. Direct and moderating links of salivary alpha-amylase and cortisol stress-reactivity to youth behavioral and emotional adjustment. Biol Psychol 2011; 88: 57-64.

7. Shankar AA, Dandekar RC. Salivary biomarkers-an update. Dent Update 2012; 39: 566-8, 571-2.

8. Castelo PM, Barbosa T de S, Pereira LJ et al. Awakening salivary cortisol levels of children with sleep bruxism. Clin Biochem 2012; 45: 651-4.

9. Monaco A, Ciammella NM, Marci MC et al. The anxiety in bruxer child. A case-control study. Minerva Stomatol 2002; 51: 247-50.

10. Barbaranelli C, Caprara GV, Rabasca A. Cuestionario “Big Five” de personalidad para niños y adolescentes. Manual. TEA Ediciones, Madrid. 2006.

11. Cortese SG, Fridman DE, Farah CL et al. Frequency of oral habits, dysfunctions, and personality traits in bruxing and non-bruxing children. A comparative study. J Cranio 2013; 31: 283-90.

12. Dworkin, SF. Research Diagnostic Criteria for Temporomandibular Disorders: current status \& future relevance. J Oral Rehabil 2010; 37: 734-43.

13. Schiffman E, Ohrbach R, Truelove E et al. Diagnostic Criteria for Temporomandibular Disorders (DC/ TMD) for Clinical and Research Applications: Recommendations of the International RDC/TMD Consortium Network* and Orofacial Pain Special Interest Group. J Oral Facial Pain Headache 2014; 28: $6-27$.

14. Timilly M, Martins C, Saulo G et al. Association between Bruxism and Salivary Cortisol Levels: A Systematic Review. Int J Odontostomat 2016; 10(3): 469-474.

15. Smith BGN, Knight JK. An index for measuring the wear of teeth. Br Dent J 1984; 156:435-438.

16. Ballerini MG, Amaro A, Otero P et al. Circadian rhythm (CR) of salivary cortisol (SAF) in children and adolescents using an ultrasensitive electrochemiluminescence immunoassay (ECLIA) 9th Joint Meeting of Paediatric Endocrinology Horm Res 2013; 80 (suppl 1) : 72 Disponible en: https://www.karger.com/ Article/Pdf/354131

17. Manfredini D, Restrepo C, Diaz Serrano K et al. Prevalence of sleep bruxism in children: a systematic review of the literature. J Oral Rehabil 2013; 40: 631-42.

18. Castroflorio T, Bargellini A, Rossini G, Cugliari G, Rainoldi A, Deregibus A. Risk factors related to sleep bruxism in children: A systematic literature review. Arch Oral Biol. 2015; 60(11): 1618-24.

19. Restrepo C, Manfredini D, Castrillon E et al. Diagnostic accuracy of the use of parental-reported sleep bruxism in a polysomnographic study in children. Int J Paediat Dent 2016; 27(5): 318-25.

20. Ohrbach R and Knibe W. Diagnostic Criteria for Temporomandibular Disorders (DC/TMD). Scoring Manual for Self-Report Instruments. ACTA, Amsterdam, The Netherlands. Jan 9, 2017. Disponible en: https://ubwp.buffalo.edu/rdc-tmdinternational/wp-content/uploads/sites/58/2017/01/DC-TMD-Selfreport-Instrument-Scoring-Manual_2017_01_09.pdf

21. Guitelman I, Farah C, Fridman D et al. Análisis del desgaste dentario en niños de 10 a 15 años. $48^{\circ}$ Reunión Científica Anual SAIO 2015. Tanti, Argentina. Disponible en: http://www.saio.org.ar/new/descargas/ Libro_XLVIII_Reunion_Cientifica_Anual.pdf

22. Maidana P, Bruno O., Mesch V. Medición de cortisol y sus fracciones: Una puesta al día. Medicina (B. Aires) 2013; 73: 579-584. 
23. Aguilar Cordero MJ, Sánchez López AM et al. Cortisol salival como indicador de estrés fisiológico en niños y adultos; revisión sistemática. Nutr Hosp 2014; 29(5): 960-968.

24. Amato, J.N.; Tuon, R.A.; Castelo, P.M. et al. Assessment of sleep bruxism, orthodontic treatment need, orofacial dysfunctions and salivary biomarkers in asthmatic children. Arc. Oral Biol 2015; 60: 698-705.

25. Oliveira MT de, Bittencourt S T, Marcon K et al. Sleep bruxism and anxiety level in children. Braz Oral Res 2015; 29: 1-5.

26. Kobayashi FY, Gavião MBD, Marquezin MCS et al. Salivary stress biomarkers and anxiety symptoms in children with and without temporomandibular disorders. Braz. Oral Res. 2017;31:e78.

27. Rashkova M, Kalchev R, Emilova L et al. Cortisol in saliva-a marker for increased anxiety in children. J IMAB - Annual Proceeding (Scientific Papers) 2010; 16(4): 67-9

28. Cruz T, Falci S, Galvao E. Association between Bruxism and Salivary Cortisol Levels: A Systematic Review. Int J Odontostomat 2016; 10: 469-474.

Recibido: $10 / 04 / 18$

Aceptado: 06/06/18

Correspondencia: Silvina Cortese. Correo: sgcortese@hotmail.com 